\title{
Friction of smooth and textured non-conformal surfaces under starved conditions
}

\author{
F. Ali, I. Křupka \& M. Hartl \\ Department of Mechanical Engineering, \\ Brno University of Technology, Czech Republic
}

\begin{abstract}
This paper presents an experimental study about the friction of non-conformal point contacts (ball-on-disk) under starved lubrication. Experiments were carried out by using a Tribometer equipped with a torque sensor for measuring the friction force and a digital camera for creating optical interferometric images. The aim of this study is to measure the friction coefficient in the sliding motion with the presence of a little amount of lubrication in the inlet of contact between the ball and disk and to investigate the effect of oil-air meniscus distance from the center of Hertzian contact on the friction between mating surfaces. In addition, the reduction of friction by artificially-produced shallow micro-dents was tested under severely starved and fully flooded conditions. Results show that the coefficient of friction increases dramatically when the air-oil meniscus starts to touch the circle of Hertzian contact and there is not such a significant difference in the friction between the starved and fully-flooded contacts since the air-oil meniscus is far away from the borders of Hertzian contact. In other words, the starvation of lubrication causes a high level of fatigue and wear of machine components when there is interference between the air-oil meniscus and the region of Hertzian contact. However, shallow micro-dents helped in reducing the friction under severe starvation conditions while the benefits of micro-dents were nearly negligible for fully flooded conditions.
\end{abstract}

Keywords: friction, textured surfaces, non-conformal, starved lubrication.

\section{Introduction}

A lot of machine components work with non-conformal point contacts such as gears, rolling-element bearings, cams etc. The pressure in such concentrated 
contacts reaches high values according to the theory of Hertz in solid contacts, so that lubrication is needed to avoid wear and metal-to-metal contact. The separation between mating surfaces depends on the film thickness of lubrication and it is well known that the EHL regime is dominated in non-conformal point contacts where the theory of EHL can successfully predict the film thickness under fully-flooded lubrication $[1,2]$. In reality it is difficult to keep the rubbing surfaces under fully flooded lubrication, e.g. in cases of severe operating condition (high speed, high load, high temperatures, start and reverse motion) or in cases where grease is used to lubricate rolling bearings. In previous cases the phenomenon of starved lubrication can be encountered where the film thickness collapses to just a few nanometers which in turn may result in component failure.

Wedeven et al. [3] used the technique of optical interferometry to investigate the phenomena of ball bearing starvation and the reduction of film thickness due to the failure of pressure build-up in the contact. The pressure in the inlet region of starved contact can be defined on the basis of a study that was performed by Elrod [4] and Elrod and Adams [5] who developed a model using the parameter $\theta$ (fractional film content) where $\theta$ represents the ratio of the oil thickness and the gap. Chiu [6] used a ball-flat rig test to study the starvation and the results obtained showed that the starvation is attributed to the insufficiency of fluid replenishment on the track. Pemberton and Cameron [7] presented a study of fluid replenishment in EHL contacts and the observation is that the balance between the entrained and the lost oil around the contact determines the position of meniscus from the Hertzian contact. Chevalier et al. [8] performed a numerical study of starved EHL point contacts and the amount of oil on the surfaces was adopted to define the degree of starvation. Recent experimental studies about the effect of starvation of point contacts on the film thickness were performed by Lubrecht et al. [9] and Cann et al. [10]. The theoretical study by Damiens et al. [11] shows that the degree of starvation depends on the operating conditions. The behavior of traction in starved EHL point contacts under rolling and sliding conditions was experimentally observed by Wedeven [12] and Querlioz et al. [13], the significance of these works is that the traction depends on the degree of starvation. Yang et al. [14] performed a numerical analysis on the traction of starved EHL line contacts and it was indicated that the traction increases rapidly at high degrees of starvation.

The proper modification of surface topography helps in reducing the friction between mating surfaces. For example, creating micro-dents on the surface, with proper dimensions and edges, reduces the interaction between asperities by emitting some amount of lubricant in the contact which results in enhancing the film thickness and pressure. On the other hand, a reduction in film thickness with deep micro-dents was investigated; see references [17-22]. Dumont et al. [23] described numerically the behavior of micro pits in the fully flooded and starved ball-on-disc contact and it was revealed that the benefits of micro pits decrease as the degree of starvation decreases because the film thickness becomes larger and the emitted amount of oil from the pits becomes negligible in comparison with the available amount in the fully flooded contact. However, a lot of efforts focused on studying the effect of micro-dents on the film thickness, while there 
is still a need to clarify the direct effect of micro-dents on the friction coefficient of non-conformal point contacts under starved lubrication.

\section{Theory}

Many factors have an influence on the friction coefficient of EHL point contacts, such as the rheological properties of lubricant, the slide-to-roll ratio, the thermal effect and so on. However, the Newtonian shear stress is given by;

$$
\tau=\eta \Delta u / h
$$

where $\tau$ is the shear stress $[\mathrm{Pa}], \eta$ the dynamic viscosity of the lubricant [Pas], $\Delta u$ velocity difference between the contacting surfaces $[\mathrm{m} / \mathrm{s}], \mathrm{h}$ the film thickness [m].

The traction force $F$;

$$
F=\int_{r_{c}=0}^{r_{c}=a} \tau d A=\int_{r_{c}=0}^{r_{c}=a}\left(\eta \frac{\Delta u}{h}\right) 2 \pi r_{c} d r_{c}
$$

where $\mathrm{F}$ the traction force $[\mathrm{N}], r_{c}$ is the radius of contact[m], $a$ is the radius of Hertzian contact $[\mathrm{m}]$.

The finite area of contact $d A$ for a circular point contact is given by;

$$
d A=2 \pi r_{c} d r_{c}
$$

The Barus equation [15], predicts the viscosities as a function of pressure by the following form;

$$
\eta=\eta_{0} \exp \left(\alpha p_{h}\right)
$$

where $\eta_{0}$ the viscosity at atmospheric pressure [Pas], $\alpha$ the pressure-viscosity coefficient $[1 / \mathrm{Pa}], \mathrm{p}_{\mathrm{h}}$ the pressure $[\mathrm{Pas}]$.

The Hertzian pressure is dominated in EHL point contacts and it is given by;

$$
p_{h}=p_{\max }\left(1-\left(r_{c} / a\right)^{2}\right)^{1 / 2}=\left(3 w \alpha / 2 \pi a^{2}\right)\left(1-\left(r_{c} / a\right)^{2}\right)^{1 / 2}
$$

If we accept that the mating surfaces are parallel in the region of Hertzian contact and the sliding velocity is constant then the coefficient of friction could be defined as;

$$
\mu=F / w=\left(2 \pi \eta_{0} \Delta u / w h\right) \int_{0}^{a} r_{c} \exp \left(\left(3 w \alpha / 2 \pi a^{2}\right)\left(1-\left(r_{c} / a\right)^{2}\right)^{1 / 2}\right) d r_{c}(6)
$$

where $\mu$ the coefficient of friction, $w$ the normal load [N].

$$
\mu=\bar{c} \bar{I} \Delta u / h
$$

where $\bar{I}=\int_{0}^{a} r_{c} \exp \left(\left(3 w \alpha / 2 \pi a^{2}\right)\left(1-\left(r_{c} / a\right)^{2}\right)^{1 / 2}\right) d r_{c}$ and $\bar{c}=2 \pi \eta_{0} / w$.

\section{Model friction and separation under starved lubrication}

Under starved conditions the shear stress in equation (1) increases with increasing the sliding velocity, which leads to increase both of the shear stress 
and the coefficient of friction. From equation (7) the coefficient of friction under starved conditions can be given as;

$$
\mu_{s}=\bar{c} \bar{I} \Delta u / h_{c s}
$$

where $\mu_{s}$ the coefficient of friction under starved conditions, $h_{c s}$ the central film thickness of starved contact[m].

Chevalier et al. [8] introduced an approximated relation representing the ratio between the central film thickness under starved conditions and the central film thickness under fully-flooded conditions by the following equation;

$$
\mathcal{R}=h_{c s} / h_{c f f}=r / \sqrt[\gamma]{1+r^{\gamma}}
$$

where the parameter $r=H_{\text {oil }} / \bar{\rho} H_{c f f}, \bar{\rho}$ is the compressibility, if it is not considered then $r=H_{\text {oil }} / H_{c f}$. The parameter $\gamma$ varies from 2 to 5. From equation (9) we can notice that the ratio $\mathcal{R}$ belongs to the range $] 0,1[$ as $r$ changes from 0 to $\infty$.

By substituting equation (9) in equation (8) we obtain the following relation;

$$
\mu_{s}=\bar{c} \bar{I} \Delta u / \mathcal{R} h_{c f f}
$$

or

$$
\mu_{s}=\mu_{f f} / \mathcal{R}=\mu_{f f} \sqrt[\gamma]{1+r^{\gamma}} / r
$$

where $\mu_{f f}$ is the coefficient of friction under given operating conditions in the fully flooded lubrication .

Under steady state conditions, the coefficient of friction $\mu_{f f}$ has a constant value and the increment of starvation degree results in a nonlinear increase in the coefficient of friction $\mu_{s}$ under the same operating conditions according to the equation (11). Figure 1 shows the simulation of equation (11).

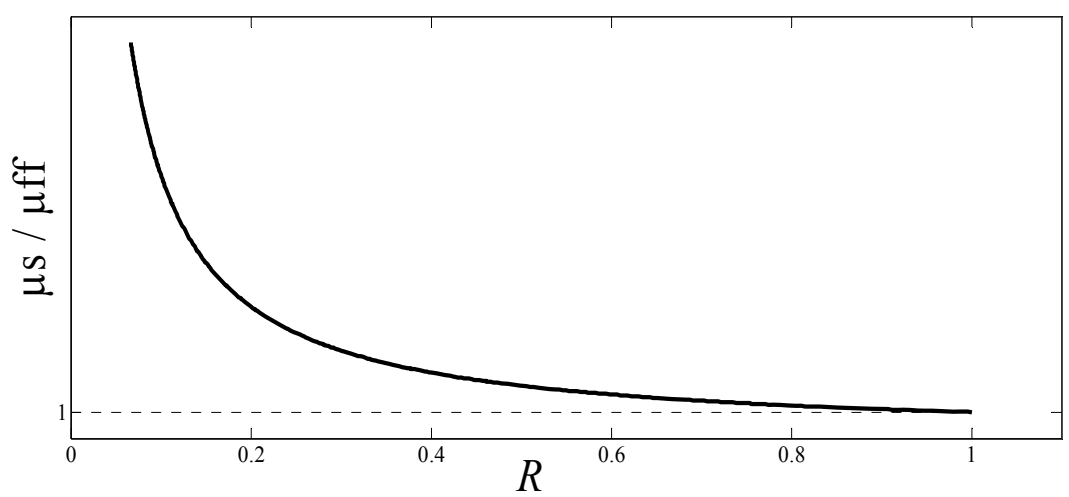

Figure 1: $\quad$ The ratio $\mu_{s} / \mu_{f f}$ versus the ratio $\mathcal{R}=h_{c s} / h_{c f f}$. 
In figure 1, two asymptotes are observed. The first asymptote shows that the ratio $\mu_{s} / \mu_{f f}$ tends to 1 as $\mathcal{R}$ tends to 1 .

$$
\lim _{\mathcal{R} \rightarrow 1}\left(\mu_{s} / \mu_{f f}\right)=1
$$

or

$$
\lim _{\mathcal{R} \rightarrow 1}\left(\mu_{s}\right)=\mu_{f f}
$$

The other asymptote shows that the ratio $\mu_{s} / \mu_{f f}$ tends to $\infty$ as $\mathcal{R}$ tends to 0 . This situation represents the friction of dry contacts or boundary regime where the starved film thickness tends to 0 and the separation is similar to the separation under boundary conditions.

$$
\lim _{\mathcal{R} \rightarrow 0}\left(\mu_{s} / \mu_{f f}\right)=\infty
$$

Under severe starved lubrication, the film thickness may diminish to a few nanometers .On the other hand, as the air-oil meniscus starts to interfere with the region of Hertzian contact, the gap between surfaces will be insufficiently filled by the fluid which inhibits the pressure build-up. If the contact is highly loaded and the pressure of the fluid in the contact is low, then a part of the load could be carried by the asperities and the regime of lubrication transforms from the EHD to the mixed lubrication ML. However the separation in the contact depends on the minimum film thickness and the roughness (RMS) of mating surfaces. Otherwise, to avoid the asperities contact under starved lubrication, the film thickness parameter $(\lambda)$ which was introduced by Tallian [16], should be larger than 1;

$$
\lambda_{s t}=h_{m s} / \sigma \geq
$$

where $\lambda_{s t}$ is the separation under starved conditions, $h_{m s}$ is the minimum starved film thickness $[\mathrm{m}], \sigma=\sqrt{R M S_{1}{ }^{2}+R M S_{2}{ }^{2}}$ where $R M S_{1}, R M S_{2}$ are the root mean square roughness of mating surfaces.

The ratio $h_{c} / h_{m}$ tends to one under starved conditions for all operating conditions. On the other hand the ratio $h_{c} / h_{m}$ tends to increase under fully flooded conditions (see reference [8]). We assume that $\mathcal{R}^{\prime}=h_{m s} / h_{m f f}$ represents the ratio between the minimum film thickness of starved and fully flooded contact, where $h_{m f f}$ is the minimum film thickness of fully flooded contact. By substituting $\mathcal{R}^{\prime}=h_{m s} / h_{m f f}$ in equation (15) we obtain:

$$
\begin{gathered}
\lambda_{s t}=\mathcal{R}^{\prime} h_{m f f} / \sigma \geq \\
\lambda_{s t}=\mathcal{R}^{\prime} \lambda_{f f} \geq 1
\end{gathered}
$$

with $\lambda_{f f}$ is the separation under fully flooded conditions. The last equation gives the conditions for keeping the contact under safe state against the metal-to-metal contact under starved conditions. Equation (17) is simulated in figure 2 for different values of $\lambda_{s t}$, where every curve in the figure represents a constant value of separation under starved lubrication conditions. Figure 2 shows that a 


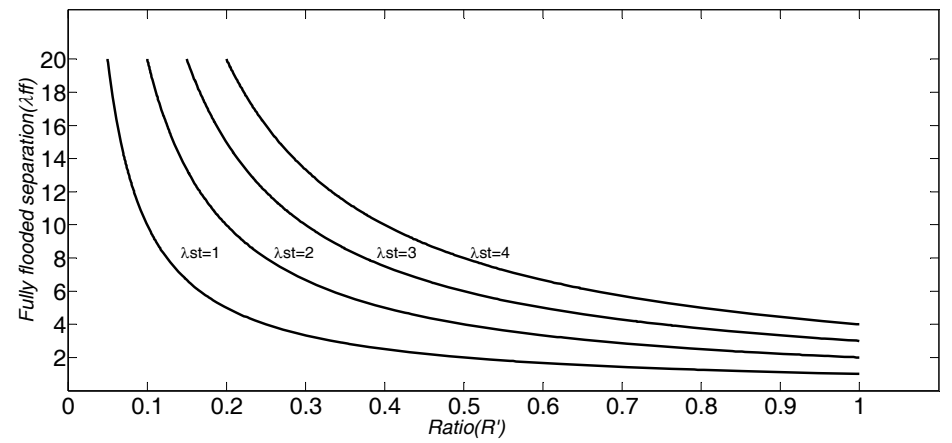

Figure 2: Relation between the separation under starved and fully flooded conditions $\left(\lambda_{s t}, \lambda_{f f}\right)$ against $\mathcal{R}^{\prime}=h_{m s} / h_{m f f}$.

constant starved separation $\left(\lambda_{s t}=\right.$ const $)$ results from a nonlinear relation between the degree of starvation and the fully flooded separation. For example, a fully flooded contact with a non-dimensional separation $\lambda_{f f}=10$ can be starved to the degree $\mathcal{R}^{\prime}=0.2$ which results in a starved separation of $\lambda_{s t}=2$. On the other hand, the same starved separation $\lambda_{s t}=2$ can be obtained from a fullyflooded separation $\lambda_{f f}=4$ with the degree of starvation $\mathcal{R}^{\prime}=0.5$. However the safe operating conditions under starved lubrication require a minimum separation larger than the height of surface asperities. This case is defined in figure 3 where the region above the curve $\lambda_{s t}=1$ represents the safe separation, whatever the degree of starvation was. On the other hand, the friction in the region above the curve $\lambda_{s t}=1$ is due to the shear stress of the lubricant (see equations (1), (6) and (7)) while the friction in the region under the curve $\lambda_{s t}=1$ is induced by the combined effect of the shear stress and the metal-to-metal contacts (see figure 3 ).

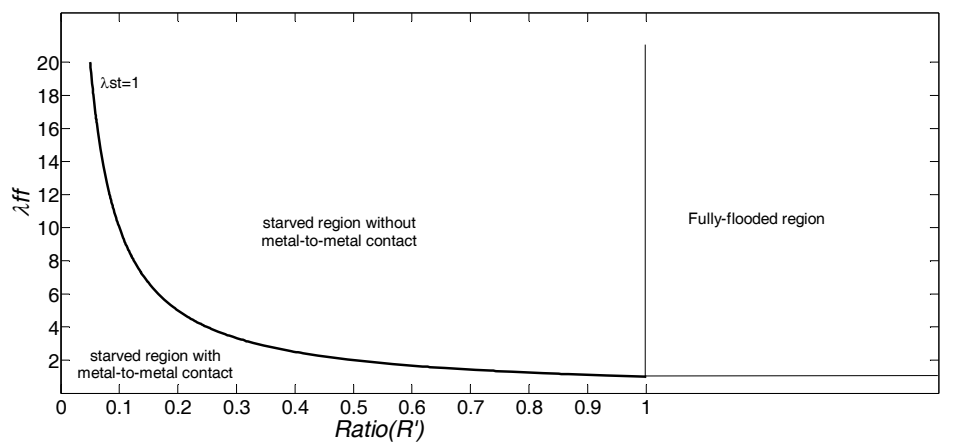

Figure 3: Distribution of starved regions with and without metal-to-metal contact. 


\section{Experimental}

A tribometer modified by adding a torque sensor on the ball shaft was used for measuring the friction force between the ball and the disk in the presence of base oil $(2400 \mathrm{~N})$ with the viscosity $\eta=0.38 \mathrm{~Pa} . \mathrm{S}$ at $40^{\circ} \mathrm{C}$. The torque sensor is integrated with a computer by using of Digital/Analog card (NI USB/6009) where the software LabVIEW receives the measurements as a digital signal to be processed and saved in the memory of the computer. The angular velocity of rotating parts is controlled by servo-motors where the velocity of ball and disk can be independently changed in the range -100 to $+100 \mathrm{rpm}$. This efficient mechanism provides the possibility of choosing the required slide-to-roll ratio under steady state and transient conditions. The diameter of the ball is 25.4 [mm] and it is made of steel AISI 52100 with a measured roughness (RMS) of about $15 \mathrm{~nm}$, while the disc is made of a transparent glass and the lower surface of the disc is coated with a thin layer of chromium. The elastic modulus of the steel ball and the glass disc is respectively $210 \mathrm{GPa}$ and $80 \mathrm{GPa}$. On the other hand, the apparatus is equipped with a digital camera (Hitachi HV-F22), the contact between the ball and disc is illuminated by a high-power source of light to improve the resolution of the images. This arrangement has the advantage of capturing interferometric images of the starved contact simultaneously with the friction measurements by the torque sensor. The amounts of lubricant for starved conditions were calibrated by a digital micropipette.

For testing the effect of micro-dents on the coefficient of friction under starved conditions, the surface of the ball was manually indented by a Rockwell indenter. 4 rows were made along the whole ball circumference. Dents were made by applying a load of $8 \mathrm{~N}$ with the illustrated distribution in figure 4, where dimensions are in $\mu \mathrm{m}$. The dent has an average diameter $35 \mu \mathrm{m}$ and a depth $0.6 \mu \mathrm{m}$.

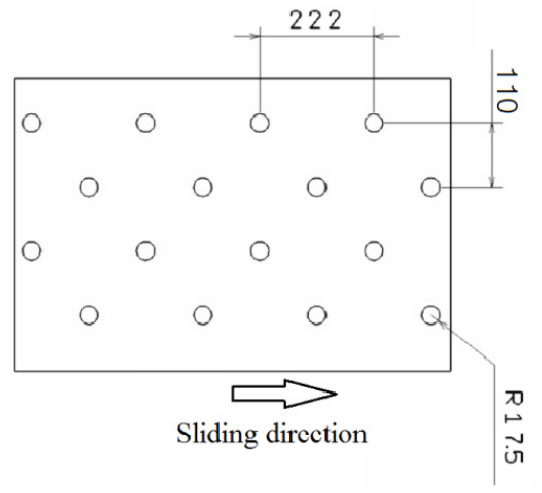

Figure 4: Distribution of micro-dents on the surface of ball. 


\section{Results}

\subsection{Stribeck curve}

The coefficient of friction was measured versus the sliding velocity under pure sliding conditions $\left(S R R=\frac{2 *\left(u_{\text {disk }}-u_{\text {ball }}\right)}{\left(u_{\text {disk }}+u_{\text {ball }}\right)}=-2\right.$, the disk is stationary $\left.u_{\text {disk }}=0\right)$ for starved contact between the ball and disc with oil amount $20 \mu \mathrm{l}$ and load $26 \mathrm{~N}$. The measurements were repeated for the fully flooded contact with the same operating conditions. The position of the air-oil meniscus was simultaneously observed by digital camera. Figure 5 shows the measured Stribeck curves for starved and fully flooded conditions and it is evident that the air-oil meniscus approaches to the center of Hertzian contact as the sliding velocity increases because the high velocities reduce the time needed to replenish the lubricant on the track, in addition to that, the high oil viscosity retards the replenishment of lubricant on the track. However, the comparison of the friction coefficient between starved and fully flooded conditions shows that the value of the friction coefficient increases strongly with increasing the sliding velocity under starved conditions, on the contrary with the fully flooded conditions where the high velocities enhance the film thickness and the separation which results in low friction. The existence of the meniscus in the vicinity of the Hertzian contact reduces the pressure build-up of fluid and inhibits the formation of film lubrication in the contact which increases the contact between the asperities and the friction. Otherwise it was observed that there is not such a large difference in the friction coefficient for starved and fully flooded conditions as the air-oil meniscus is far enough from the center of Hertzian contact at low sliding velocities.

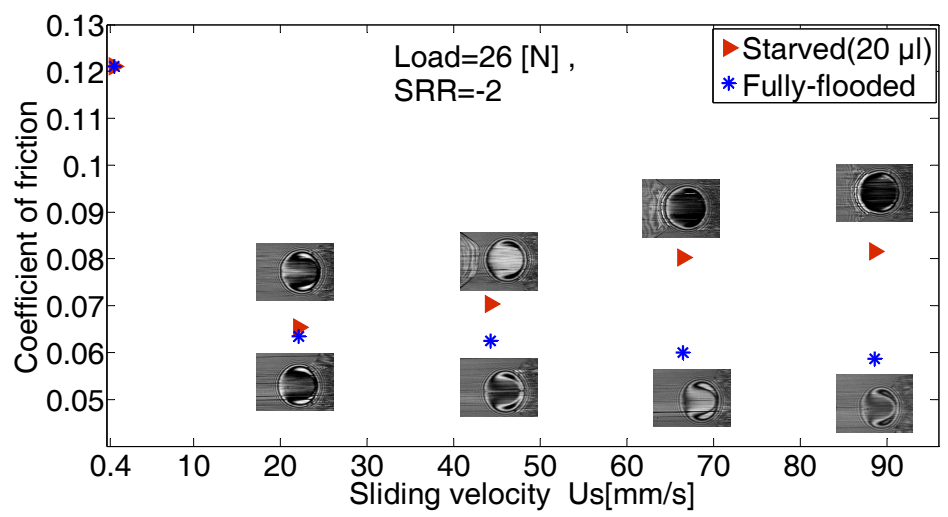

Figure 5: Comparison of Stribeck curves for lubricated point contacts under starved and fully-flooded conditions. 


\subsection{Steady state}

The effect of air-oil meniscus position was observed under steady state operating conditions of the sliding velocity and load ( $\mathrm{SRR}=-1$, entraining velocity $u_{e}=$ $30[\mathrm{~mm} / \mathrm{s}]$, load $=32 \mathrm{~N}$ ) where the degree of starvation was modified by changing the oil amount on the track (see figure 6 and please note that the images were captured in the 5th minute). Interferometric images show that the coefficient of friction increases largely under starved conditions when the air-oil meniscus starts to touch the Hertzian contact with only a small amount of lubricant $(14 \mu \mathrm{l})$ on the track. Otherwise, the coefficient of friction of starved and fully flooded contact is nearly the same for a sufficient amount of oil $(42 \mu \mathrm{l})$ on the track. The sudden rise of the friction coefficient is attributed to the insufficient replenishment of fluid on the track for the low amount of lubricant which results in reducing the distance between the air-oil meniscus and the center of Hertzian contact.

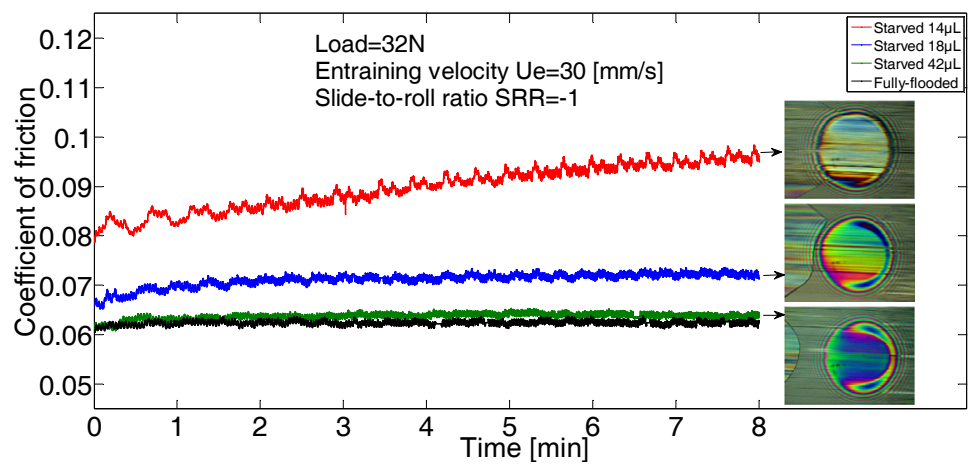

Figure 6: Effect of oil amount on the coefficient of friction and the position of air-oil meniscus.

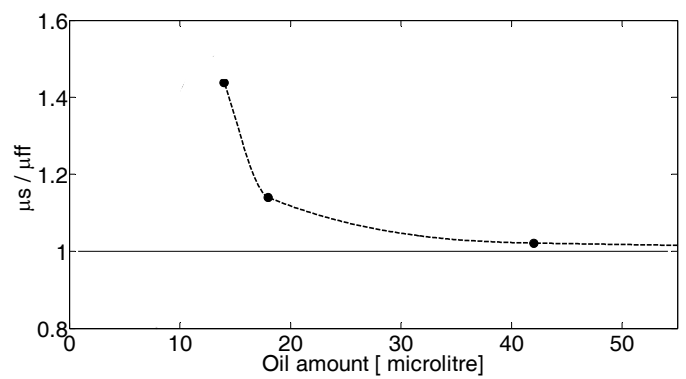

Figure 7: Experimental relation between the ratio $\mu_{s} / \mu_{f f}$ and the oil amount on the track.

The experimental relation between the ratio $\mu_{s} / \mu_{f f}$ and the amount of oil is represented in figure 7 where the values of $\mu_{s}$ and $\mu_{f f}$ were calculated as the 
mean value during 8 minutes. The ratio $\mu_{s} / \mu_{f f}$ tends to one as the degree of starvation reduces, however this result is in accord with figure 1 and equation (12).

\subsection{Friction of textured surfaces}

In order to investigate the effect of micro-dents in reducing the friction, the surface of the ball was artificially modified by 4 rows of shallow micro-dents, see figure 8 . The average diameter of the dent is about $35 \mu \mathrm{m}$ with a depth of about $0.6 \mu \mathrm{m}$. Figures 9 and 10 show a comparison of the friction coefficient for

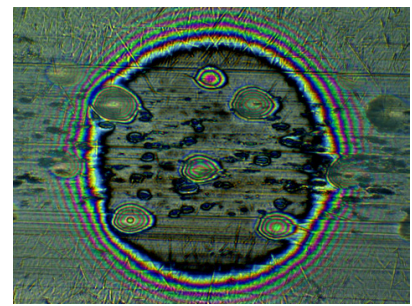

Figure 8: Micro-dents on the ball surface.

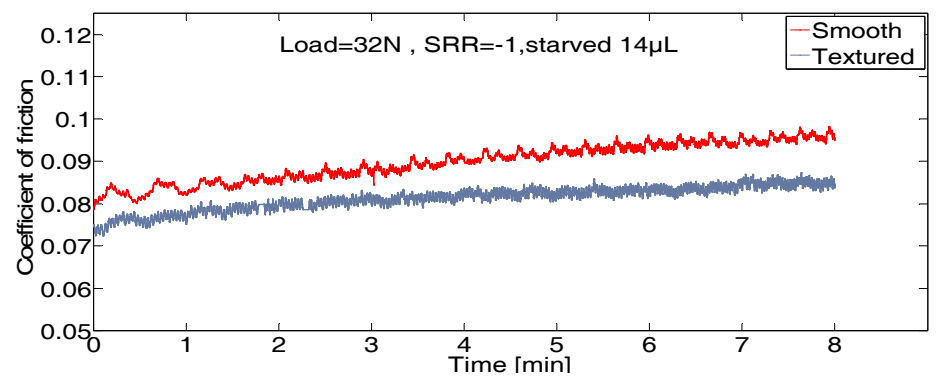

Figure 9: Coefficient of friction for smooth and textured surfaces under starved conditions.

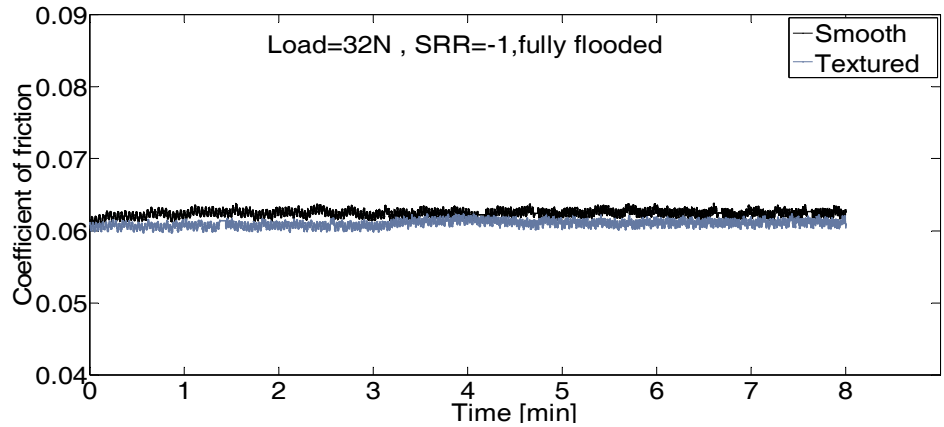

Figure 10: Coefficient of friction for smooth and textured surfaces under fully flooded conditions. 
smooth and textured surface under starved and fully flooded lubrication. The results show that the benefits of micro-dents under starved lubrication are proportionally considered where the reduction of friction is about $9 \%$. On the other hand, the benefits of micro-dents under fully flooded conditions are negligible and the value of average friction of smooth and textured surfaces is nearly the same; this is justified by the fact that the amount of emitted fluid from the micro-dent doesn't make a noticeable difference in enhancing the film thickness under fully flooded lubrication where the film is originally large enough.

\section{Conclusions}

The friction coefficient of a non-conformal EHL point contact was measured under starved and fully flooded conditions and the benefits of surface texturing were also investigated. Results show that the friction coefficient of nonconformal point contacts increases dramatically and non-linearly under starved conditions when the sliding velocity increases; this action is accompanied by reducing the time to replenish the lubricant on the track which results in minimizing the distance between the air-oil meniscus and the center of Hertzian contact. Under steady state conditions, the friction coefficient depends strongly on the oil amount on the track. On the other hand; the value of the friction coefficient was almost the same for fully flooded and starved contact when the air-oil meniscus was far away from the Hertzian contact. The modification of non-conformal mating surfaces by shallow micro-dents provided a considerable reduction of friction (about 9\%) under severe starved lubrication. Otherwise the benefits of micro-dents were negligible for fully flooded lubrication.

\section{References}

[1] Hamrock, BJ.; Dowson, D., 1977, "Isothermal elastohydrodynamic lubrication of point contacts. Part III-fully flooded results", J Lubr Technol, Vol.99, pp. 264-76.

[2] Dowson, D.; Higginson, GR., 1959, "A numerical solution to the elastohydrodynamic problem”, J Mech Eng Sci, Vol.1, pp. 6-15.

[3] Wedeven, L.D.; Evans, D.; Cameron, A. C., 1971, "Optical Analysis of Ball Bearing Starvation”, ASME J. Lubr. Technol, Vol. 93, pp. 349-363.

[4] Elrod, H. G., 1981, “A Cavitation Algorithm”, ASME J. Lubr. Technol, Vol. 103, pp. 350-354.

[5] Elrod, H. G.; Adams, M. L., 1974, "A Computer Program for Cavitation and Starvation Problems", Proceedings of the 1st Leeds-Lyon Symposium on Tribology, pp. 37-41.

[6] Chiu, Y. P., 1974, "An Analysis and Prediction of Lubricant Film Starvation in Rolling Contact Systems", ASLE Trans, Vol.17, pp. 22-35.

[7] Pemberton, J.; Cameron, A. C., 1976, "A Mechanism of Fluid Replenishment in Elastohydrodynamic Contacts", Wear, Vol.37, pp. 185190. 
[8] Chevalier, F.; Lubrecht, A. A.; Cann, P. M. E.; Colin, F.; Dalmaz, G., 1998, "Film Thickness in Starved EHL Point Contacts", ASME J. Tribol, Vol.120, pp. 126-133.

[9] Lubrecht, T.; Mayuzer, D.; Cann, P., 2001, "Starved Elastohydrodynamic Lubrication Theory: Application to Emulsions and Greases", Comptes Rendus de l'Academie des Sciences. Serie IV, Physique, Astro- physique, 2(5), pp. 717-728.

[10] Cann, PME.; Damiens, B.; Lubrecht, A.A., 2004, “The transition between fully flooded and starved regimes in EHL", Tribol Int, Vol.37, pp. 859-64.

[11] Damiens, B.; Venner, C.H.; Cann, P.M.E.; Lubrecht, A.A., 2004, "Starved lubrication of elliptical EHD contacts”, ASME J. Tribol, Vol. 126, pp. 105111 .doi:10.1115/1.1631020.

[12] Wedeven, L.D., 1975, "Traction and film thickness measurements under starved elastohydrodynamic conditions", Trans. ASME, J. Lubric. Technol, Vol. 97, pp. 321-329.

[13] Querlioz, E.; Ville, F.; Lenon, H.; Lubrecht, T., 2007, "Experimental Investigations on the Contact Fatigue Life under Starved Conditions", Tribol Int, Vol.40, pp. 1619-1626.

[14] Yang, P.; Wang, J.; Kaneta, M., 2006, "Thermal and Non-Newtonian Numerical Analyses for Starved EHL Line Contacts", ASME J. Tribol., Vol. 128, pp. 282-290.

[15] Barus, C., 1893, "Isothermals, Isopiestics and Isometrics Relative to Viscosity", American Journal of Science, Vol.45, pp. 87-96.

[16] Tallian, T.E., 1967, "On Competing Failure Modes in Rolling Contact", ASLE Transactions, Vol. 10, pp. 418-439.

[17] Nakatsji, T.; Mori, A., 2001 "The tribological effect of mechanically produced micro-dents by a micro diamond pyramid on medium carbon steel surfaces in rolling-sliding contact", Meccanica, Vol. 36, pp. 663-674.

[18] Coulon, S.; Jubault, I.; Lubrech, A.A.; Ville, F.; VERGNE, P., 2004, "Pressure profiles measured within lubricated contacts in presence of dented surfaces. Comparison with numerical models", Tribology International, Vol. 37, pp. 111-117.

[19] Nelias, D.; Ville, F., 2000, "Detrimental effects of debris dents on rolling contact fatigue", Journal of Tribology-Transactions of the Asme, Vol. 122, pp. 55-64.

[20] Mourier, L.; Mazuyer, D.; Lubrecht, A.A.; Donnet, C., 2006, "Transient increase of film thickness in micro-textured EHL contacts", Tribology International, Vol. 39, pp. 1745-1756.

[21] Ai, X.L.; Cheng, H.S., 1994, "The influence of moving dent on point EHL contacts", Tribology Transactions, Vol. 37, pp. 323-335.

[22] Krupka, I.; Hartl, M., 2007, "The effect of surface texturing on thin EHD lubrication films", Tribology International, Vol. 40, pp. 1100-1110.

[23] Dumont, M.; Lugt, P.M.; Tripp, J.H., 2002, "Surface feature effects in starved circular EHL contacts", Journal of Tribology-Transactions of the Asme, Vol. 124, pp. 358-366. 